\title{
PHYSIOLOGICAL MENSTRUAL RHYTHM AND FERTILITY AFTER INTERNAL ILIAC ARTERY LIGATION
}

\author{
Sunaina Srinivasan ${ }^{1}$, Nidhi Sharma ${ }^{2}$, K. Jayashree $^{3}$
}

${ }^{1}$ Senior Resident, Department of Obstetrics and Gynaecology, Institute of Obstetrics and Gynaecology, Madras Medical College, Chennai.

${ }^{2}$ Associate Professor, Department of Obstetrics and Gynaecology, Saveetha Medical College, Saveetha University, Chennai. 3 Professor and HOD, Department of Obstetrics and Gynaecology, Saveetha Medical College, Saveetha University, Chennai.

\section{ABSTRACT}

\section{AIMS AND OBJECTIVE}

Bilateral internal iliac artery ligation is a useful skill for the management of life-threatening post-partum hemorrhage. The procedure is not without risks and long-term complications. This study was conducted to ensure documentation and reporting of pregnancies following bilateral hypogastric artery ligation in cases of post-partum hemorrhage. We also aimed to compare the menstrual function and reproductive outcome after bilateral internal iliac artery ligation done for atonic post-partum haemorrhage.

\section{DESIGN}

Prospective Case Study.

\section{SETTING}

Department of Obstetrics and Gynaecology, Institute of Obstetrics and Gynaecology, Madras Medical College and Hospital, Chennai, Jan 2013 to March 2015.

\section{PARTICIPANTS}

Ten cases of bilateral internal iliac artery ligation were done for atonic post-partum hemorrhage.

Main outcome measures: The primary outcome was assessed as the resumption of menstruation, regular cycles, and ability to conceive after surgery. Secondary outcome was incidence of intrauterine growth retardation and recurrent post-partum hemorrhage in subsequent pregnancies.

\section{RESULTS}

The mean duration of resumption of menstruation following internal iliac artery ligation was 3 months. Bilateral internal iliac artery ligation is preferred over unilateral ligation as the extensive collaterals from the contralateral side immediately fill the circulation. The cases of atonic post-partum hemorrhage may require B-Lynch sutures in addition to bilateral internal iliac artery ligation to regain tone. Successful pregnancy after bilateral internal iliac artery ligation occurred in three out of seven cases. In one case, there was intrauterine growth retardation in eighth month. The patient was admitted and closely monitored with Doppler scan. At 35 weeks of gestation, emergency lower segment caesarean section was done for absent diastolic flow. The preterm neonate was 2 kilograms. There was no post-partum hemorrhage in the subsequent gestation.

\section{CONCLUSION}

Bilateral internal iliac ligation is a safe procedure in trained hands. Most near miss cases of maternal mortality have been saved due to this life saving surgery. Modern specialization has resulted in loss of art in the practice of general gynecology. The surgery is simple and should be within the surgical expertise of main stream gynecology.

\section{KEYWORDS}

Internal Iliac Artery Ligation, Menstruation, Fertility.

HOW TO CITE THIS ARTICLE: Sunaina Srinivasan, Nidhi Sharma, K. Jayashree. "Physiological Menstrual Rhythm and Fertility After Internal Iliac Artery Ligation." Journal of Evolution of Medical and Dental Sciences 2015; Vol. 4, Issue 96, November 30; Page: $16147-16149$, DOI: $10.14260 /$ jemds/2015/2368

\section{INTRODUCTION}

The internal iliac artery ligation was pioneered by Harward Kelly.(1) to control intraoperative bleeding from cervical cancer. Bilateral internal iliac artery ligation is a lifesaving procedure in post-partum hemorrhage. In most near miss cases of maternal death, bilateral internal artery ligation along with aggressive intravenous

Financial or Other, Competing Interest: None.

Submission 28-09-2015, Peer Review 29-09-2015,

Acceptance 20-11-2015, Published 27-11-2015.

Corresponding Author:

Dr. Nidhi Sharma,

No. 5, Jayanthi Street,

Velachery-600042,

Chennai.

E-mail: drbonuramkumar@yahoo.co.in

DOI:10.14260/jemds/2015/2368 fluid therapy, uterotonics, uterine massage, early intubation and inotropics save the patient.(2)

This requires a tertiary care set up and surgical skills for retroperitoneal ligation of internal iliac artery ligation. ${ }^{(3)}$ Internal iliac artery ligation can lead to immediate and remote complications. Immediate complications include injury to common iliac artery, external iliac artery or vein, ureter or obturator nerve. The injury can occur because of mistaken identification. Long term complications include foot drop due to accidental incorporation of anterior division of the sciatic nerve, bladder ischemia, gluteus muscle ischemia, amenorrhea and infertility.

An advantage of bilateral internal iliac artery ligation over obstetrical hysterectomy is the preservation of reproductive function. In obstetrical hysterectomies the uterine artery is clamped on both sides; while in bilateral ligation of internal iliac artery the visceral branches clamped are the uterine, the superior vesical artery, the middle vesical 
artery, the inferior vesical artery, the middle haemorrhoidal artery, the inferior haemorrhoidal artery and vaginal artery

The parietal branches secured are the obturator artery, the inferior gluteal artery and the internal pudendal artery. This leads to reduction of pulse pressure (Systolic pressure diastolic pressure) in the pelvis. The internal iliac artery distill to the point of ligation is never emptied of blood. The collateral circulation takes over immediately. However, the trip hammer effect of arterial pulsations is stopped and the arterial pumping system is converted to venous drainage conduits. The reduced blood pressure although lead to a theoretical risk of venous thrombosis, the blood supply to uterine endometrium may be compromised. There may be an impaired return of fertility as the signal mediators of Hypothalamic-pituitary-ovarian-endometrial axis (FSH, LH, estrogens, progesterone) are transmitted through blood.

An additional risk in an exsanguinated hypovolemic patient of post-partum hemorrhage is pituitary apoplexy. The necrosis of gonadotropin secreting cells of anterior pituitary leads to permanent amenorrhea and infertility (Sheehan's syndrome). There are case reports of normal pregnancy and delivery occurring after internal iliac artery ligation, but a strict followup is essential to document the safety. Hence, this study was conducted to ensure documentation and reporting of pregnancies following bilateral hypogastric artery ligation in cases of post-partum hemorrhage.

\section{MATERIALS AND METHODS}

This prospective study was done in our teaching institute from January 26, 2013 to 31 March, 2015, after Institutional Review Board Approval. Ten case of atonic post-partum hemorrhage following lower segment caesarean section were studied. Seven patients were primigravidas and three were multigravidas. In three patients of atonic post-partum haemorrhage, B-Lynch sutures were also applied.(4) along with bilateral internal iliac ligation. In three multigravidas, concurrent bilateral tubal sterilization was done. All cases of bilateral internal iliac artery ligation were followed for resumption of menstrual rhythm.

\section{SURGICAL TECHNIQUE}

The landmark of bifurcation of common iliac artery to internal iliac artery and external iliac artery is a plane drawn through sacral promontory and bilateral anterior superior iliac spines.(5) Visibility is remarkable better if the surgeon changes sides while operating on the contralateral vessels. The peritoneal incision begins horizontally behind the mesentery of round ligament at junction of its medial and middle third. The peritoneal incision is carried horizontally on the lateral pelvic wall for $10 \mathrm{~cm}$. The peritoneal flaps on the medial side have the ureter attached to the peritoneum.

The ureter crosses the common iliac artery from lateral to medial side just above the bifurcation. The lateral flap of peritoneum hides the external iliac artery and vein overlying the psoas major and minor muscles. The internal iliac artery is seen arising at right angles from the bifurcation and the straight continued artery is the external iliac, which continues as femoral artery in the leg.

Pledgets like used in dental surgery are used to clear the loose areolar tissue in the direction of vessels. Once the vessels are identified, a right angle clamp is gently and firmly inserted between the internal iliac artery and internal iliac vein from lateral-to-medial side (To avoid the injury to external iliac vessels which lie laterally). Two suture of non- absorbable silk are tied $0.5-1 \mathrm{~cm}$ apart on the anterior division around $1 \mathrm{~cm}$ below the internal iliac artery gives its posterior branch (Figure 1). Transection of internal iliac artery anterior division between the two ligatures is unnecessary and leads to inadvertent injury to internal iliac vein. It is better avoided.

Before closing the abdomen, a thorough check is made of femoral pulse through a hand held Doppler. The posterior peritoneum may or may not be closed. If peritoneal closure is contemplated, it should be intermittent and careful to prevent ureteric injuries. If damage to iliac veins happen, the immediate reflex should be to apply firm pressure both below and above the site of injury. Two suction cannulas may be used to visualize the operative site adequately. When the defect in iliac vein is seen, the two edges of tear should be held with atraumatic stiles forceps. A non-absorbable 30 polypropylene is used to repair the vein. If the rent in internal iliac vein is not visualized, the internal iliac artery may be transected between the two sutures for better visualization. The vagina was checked for excessive bleeding before closing the peritoneum.

\section{DISCUSSION}

The mean duration of resumption of menstruation following internal iliac artery ligation was 3 months. Bilateral internal iliac artery ligation is preferred over unilateral ligation as the extensive collaterals from the contralateral side immediately fill the circulation. The cases of atonic post-partum hemorrhage may require B-Lynch sutures in addition to bilateral internal iliac artery ligation to regain tone.(4) Successful pregnancy after bilateral internal iliac artery ligation occurred in three out of seven cases. In one case, there was intrauterine growth retardation in eighth month.

The patient was admitted and closely monitored with Doppler scan of uterine artery (Figure 2). At 35 weeks of gestation, emergency lower segment caesarean section was done for absent diastolic flow. The preterm neonate was 2 kilograms. There was no post-partum hemorrhage in the subsequent gestation.

Likely causes of failure of this procedure include large anomalous vascular supply of the uterus, massive necrosis of vessels in exsanguinated patients and concurrent venous hemorrhage. If disseminated vascular coagulation is persisting despite adequate platelet and fibrinogen transfusion, the surgery fails to control bleeding.

\section{COMPLICATIONS}

There were no injuries to external iliac vessels, internal iliac vein and ureter in our series of bilateral internal iliac ligation cases. In earlier literature, case reports of buttock skin necrosis have been cited.(6)

\section{CONCLUSION}

Our study suggests that bilateral internal iliac artery ligation carries a considerable safety as in other studies.(7) and future fertility is not compromised when proper patient selection, intraoperative techniques and post-operative care is provided. The resumption of menstrual rhythm is established in 3 months. Future fertility is not compromised and there is no risk of recurrent post-partum hemorrhage. The subsequent pregnancies should be monitored for intrauterine growth retardation with intensive bi-weekly fetal Doppler of umbilical and middle cerebral arteries. 


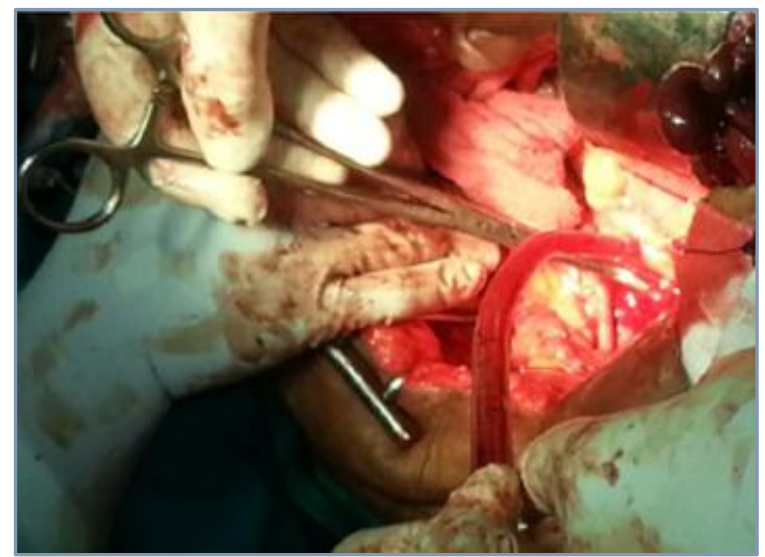

Figure 1: The clamp is inserted from lateral-to-medial side. The posterior division of internal iliac is identified. The anterior division of internal iliac is ligated with Mersilk. Transection is should not to be done.

\section{REFERENCES}

1. Kelly $H$. Ligation of both internal iliac arteries for hemorrhage in hysterectomy for carcinoma uteri. Bull John Hopkins 1894;5-53.

2. Varner Obstetrical Emergencies (Post-partum haemrrhage). Crit Care 1991;7:883-97.

3. Burchell R C. Internal iliac artery ligation: Aortograms. American Journal of obstet gynaecol 1966;94:117.

4. Lynch C, Coker Y, Abu J, et al. The B-Lynch surgical technique for the control of massive post-partum hemorrhage: an alternative to hysterectomy? Five case reported. Br J Obstet Gynaecol 1997;104:372-5.

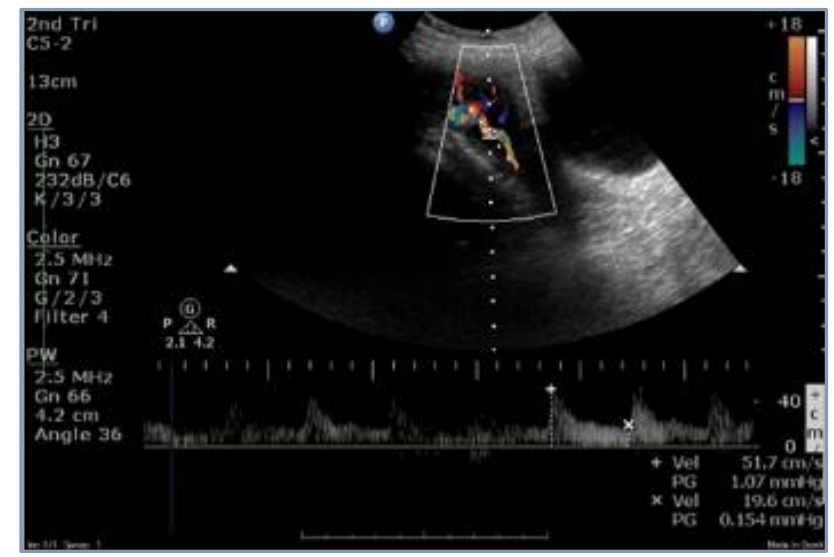

Figure 2: In Second Trimester Doppler study of uterine artery was used to monitor pregnancies following internal iliac artery ligation.

5. Reich WJ, Nechtow HJ, Bogdan J. The iliac arteries: a gross anatomical study based on dissection of 75 fresh cadavers. Clinical Surgical correlations. J Int Coll Surg 1964;41:53.

6. Tejas RV. Ligation of the hypogastric arteries and its complications in resection of cancer of rectum. Am J of Gastroenterology? 1956;26:612.

7. Burschell RC, Mengret WF. Internal iliac artery ligation: a series of 200 patients. J Int Fed Obstet Gynaecol 1969;41:53. 